\title{
Levels and Associations of Weight Misperception with Healthy Lifestyle among Adults in Saudi Arabia
}

\author{
Nora A. Althumiri ${ }^{a}$ Mada H. Basyouni ${ }^{a, b}$ Nasser F. BinDhim ${ }^{a, c, d}$ \\ Saleh A. Alqahtani $\mathrm{i}^{\mathrm{e} f}$ \\ aSharik Association for Health Research, Riyadh, Saudi Arabia; ${ }^{b}$ Ministry of Health, Riyadh, Saudi Arabia; ${ }^{c}$ College \\ of Medicine, Alfaisal University, Riyadh, Saudi Arabia; dSaudi Food and Drug Authority, Riyadh, Saudi Arabia; \\ eLiver Transplant Unit, King Faisal Specialist Hospital \& Research Centre, Riyadh, Saudi Arabia; ${ }^{\circ}$ Division of \\ Gastroenterology and Hepatology, Johns Hopkins University, Baltimore, MD, USA
}

\section{Keywords}

Body mass index · Obesity · Weight misperception · Weight misclassification · Saudi Arabia

\begin{abstract}
Introduction: Obesity affects more than one-quarter of adults in Saudi Arabia and is on the rise. A significant proportion of overweight and obese individuals misperceive their weight, and this misperception can affect their likelihood to exercise, consume healthy foods, or change unhealthy dietary habits. This study examines the prevalence of weight misperceptions in a national sample of Saudi adults and explores weight misperception association with sociodemographic factors, behavioral factors, and health status. Methods: This study was a nationwide cross-sectional survey conducted via phone interviews. A proportional quota sampling technique was used to obtain equal distributions of participants, stratified by age and gender, across the 13 regions of Saudi Arabia. Weight and height were self-reported, and obesity was determined as a BMI $\geq 30$. Participants were
\end{abstract}

asked to describe their current weight as ideal, underweight, overweight, or obese. Misclassification was then compared across groups according to a range of sociodemographic factors, behavioral risk factors, and health indicators. $\boldsymbol{R e}$ sults: Of the 6,239 participants contacted, 4,709 (75.5\%) responded and completed the interview. Of them, $50.1 \%$ were female, and the mean age was 36.4 (SD \pm 13.55 ) years. The majority $(70.1 \%)$ of participants indicated having weighed themselves within the past 30 days. The prevalence of weight misperception in general was $42.0 \%$, and in obese participants, it was $67.6 \%$. In addition, a significant association was observed between weight misperception and obesity, agegroup, educational level, diagnosed chronic condition, selfrated health, and sedentary lifestyle. There was no significant association between weight misperception and gender, physicalactivity, or a nutritional knowledge.Conclusions: Weight misperception could be used as an indicator of a poor health routine that may lead to negative health outcomes.
(C) 2021 The Author(s)

Published by S. Karger AG, Basel karger@karger.com www.karger.com/ofa

Karger $\stackrel{\text { ' }}{5}$

BOPEN ACCESS
(C) 2021 The Author(s)

Published by S. Karger AG, Basel

This is an Open Access article licensed under the Creative Commons Attribution-NonCommercial-4.0 International License (CC BY-NC) (http://www.karger.com/Services/OpenAccessLicense), applicable to the online version of the article only. Usage and distribution for commercial purposes requires written permission.
Correspondence to:

Nora A. Althumiri, nora@althumiri.net 


\section{Introduction}

Overweight and obesity are major public health concerns worldwide, with prevalence being historical high [1]. The World Health Organization (WHO) estimates that roughly $39 \%$ of all adults are overweight, while $15 \%$ of women and $11 \%$ of men are obese [2]. In Saudi Arabia, around $25 \%$ of the population is obese [3-5]. Obesity is a significant risk factor for a range of physical and mental health problems, including cardiovascular disease, type 2 diabetes, heart attack, stroke, and depression [6,7]. Obesity also impacts well-being, by increasing depression, body dissatisfaction, binge eating, and the use of unhealthy weight control behaviors [8-11]. Given its substantial effects on physical and mental health and well-being, obesity remains one of the most pressing health priorities of current times.

For weight loss interventions to be successful, people need to be aware of their actual weight. However, there are a subset of individuals who are misperceiving their actual weight status, which may in turn affect adult's weight management behaviors. Weight misperception defined to be the over- or underestimation of one's weight can itself be a barrier to health-related improvements [12]. A recent study conducted in Korea has found that $49.3 \%$ of Korean youth misperceived their weight status, with similar prevalence of over- and underestimation. Nonoverweight adults who overestimate their weight may experience body dissatisfaction, leading to a greater risk for eating disorders. Contrarily, overweight adults who misperceive their weight may be less motivated to lose weight; thus, it may increase chronic disease risk and become less inclined to engage in physical activity or consume a healthy diet $[13,14]$.

Sociodemographic factors may play a role in weight misperception. Women appear to be at a greater risk of weight misperception, weight dissatisfaction, and disordered eating than their male counterparts [15]. Age has also been implicated in the likelihood of weight misperception, with older overweight and obese people more likely to underestimate their weight status than those who are younger [16].

Further, prevalence estimates for weight misperception have been found to vary substantially between countries. The proportions of weight misperception in the USA and Canada are approximately $20 \%$ and $30 \%$ among men and women $[17,18]$, respectively, while a recent Korean study found that approximately $40 \%$ of the population incorrectly reported their weight status [19]. Although prevalence estimates from large, national surveys in Saudi Arabia are lacking, a recent study of Saudi med- ical students reported that weight misperception occurred in nearly $64 \%$ of participants - a substantially higher rate than other countries [20]. Differences in sociocultural background and rates of obesity may play a role in the differences observed between countries [16].

Limited data exist on the prevalence of weight misperception in Saudi Arabia, and population-level data on the relationship between weight misperception and obesity and socioeconomic factors are currently lacking. Further, most local studies of weight perception involve either targeted experiments of select individuals (e.g., lean and obese people) or surveys conducted in specific subpopulations (e.g., college students and children). A better understanding of the factors associated with weight misperception in Saudi adults has the potential to improve health promotion efforts by identifying groups most vulnerable to misperception and its consequent effects.

Therefore, the aim of the current study was to build upon the existing research on weight misperception in Saudi Arabia and extend these findings to the national level using a larger, more representative sample. We further sought to explore the relationship between weight misperception for overweight and obese participants with sociodemographic factors, lifestyle factors, and health status.

\section{Method}

Study Design

This study was a nationwide, cross-sectional survey conducted in Saudi Arabia by phone interviews in June 2020.

Sampling Method and Sample Size

A proportional quota sampling technique was employed to acquire an equal distribution of participants stratified by age and gender across the 13 regions of Saudi Arabia. Two age-groups $(\geq 36$ and $<37$ ) based on the median age of Saudi Arabian adults (36 years old) were used, leading to 52 quotas (4 quotas per region: male 18-36 years, male $37+$ years, female $18-36$ years, female $37+$ years). The QPlatform data collection system, which has integrated eligibility and sampling modules, was used to control the sample distribution [21]. The eligibility module included 3 questions to determine the completeness of the sampling quota, including age, gender, and region. The sample size was calculated to provide sufficient statistical power to compare between any 2 quotas on a medium effect size of approximately 0.25 using (Cohen [22]) effect size tables for categorical variables ( $80 \%$ power, $95 \% \mathrm{CI}$ ) to compare age and gender across regions. Each quota required 90 participants, and the total target sample comprised 4,680 participants.

\section{Participant Recruitment}

Participant recruitment was limited to Arabic-speaking Saudi residents aged 18 years and older. A phone number list was randomly generated from the Sharik Association for Health Research 
to identify potential participants [23]. The Sharik database includes individuals who are interested in participating in research projects and contains a growing number of registered participants $(>73,000)$ distributed across the 13 regions of Saudi Arabia [23]. Participants were contacted by phone on up to 3 occasions by trained interviewers. If they did not respond, then a new phone number for a person with similar demographics was generated from the database until the quota was completed and automatically closed. After obtaining consent to participate, the interviewer assessed participant eligibility using the quota completion criteria described before.

\section{Survey and Outcome Measures}

The survey questions were adopted from the 2016-2017 National Health and Nutrition Examination Survey (NHANES) [24]. The survey included questions addressing sociodemographic information (age, gender, education, and region), behavioral risk factors (nutritional knowledge, physical activity, and sedentary lifestyle), and health status (chronic physical conditions and selfrated health).

Basic nutritional knowledge was assessed by asking the participants 4 true-or-false questions developed for this study. (1) The first question was "Sugar should be the main source of calories"; (2) "the portion size is the total number of calories that come from carbohydrates and protein"; (3) "protein is high in sugar, which is why it is recommended to lower your intake of it"; and (4) "to lose weight, generally it recommended to consume $>3$ portions of fruits every day." Then, the score was tabulated from 0 to 4 based on how many correct answers the participants could provide. Physical activity was self-reported as the number of days per week a participant performed at least 30 min of moderate intensity physical activity or the number of days a participant performed 20 min or more of vigorous physical activity in the previous 2 weeks. The WHO criteria were then applied to categorize physical activity as moderate (150 min/week of moderate physical activity) or vigorous ( $75 \mathrm{~min} /$ week of vigorous physical activity). Sedentary lifestyle was measured as the number of hours per day of leisure sitting, such as while watching television, using a mobile phone, or playing video games. Sedentary lifestyle was grouped into 4 categories ranging from $<2 \mathrm{~h}$ per day to $>6 \mathrm{~h}$ per day. Self-rated health was assessed on 5-point Likert scale ranging from 1 (poor) to 5 (excellent). Chronic health conditions were assessed by asking the participants if they were diagnosed with the disease and currently on treatment.

BMI was calculated from self-reported height and weight; to increase the quality of the self-reported weight, we asked the participants the last time they measured their weight. BMI then categorized into 4 groups: underweight $\left(\leq 18.5 \mathrm{~kg} / \mathrm{m}^{2}\right)$, normal weight $\left(18.5-24.9 \mathrm{~kg} / \mathrm{m}^{2}\right)$, overweight $\left(25-29.9 \mathrm{~kg} / \mathrm{m}^{2}\right)$, and obese $(30 \mathrm{~kg} /$ $\mathrm{m}^{2}$ and above). Weight misperception was measured using the question: do you consider yourself now to be (underweight, overweight, or about the right weight)? Linguistic validation was performed via a focus group of 7 participants from the general population to ensure that the translation of the original questionnaire from English to Arabic conveyed the same information and intention.

Primary Outcomes of Interest

The primary outcomes we sought to understand were the proportion of adults who misperceived weight and the relationship
Table 1. Sociodemographic characteristics, behavioral risk factors, and health status of participants

\begin{tabular}{|c|c|}
\hline Variable & Proportion, $n(\%)$ \\
\hline \multicolumn{2}{|l|}{ Age-groups, years } \\
\hline $18-19$ & $255(5.4)$ \\
\hline $20-29$ & $1,556(33.0)$ \\
\hline $30-39$ & $1,009(21.4)$ \\
\hline $40-49$ & $1,044(22.2)$ \\
\hline $50-59$ & $555(11.8)$ \\
\hline $60+$ & $290(6.2)$ \\
\hline \multicolumn{2}{|l|}{ Gender } \\
\hline Female & $2,358(50.1)$ \\
\hline Male & $2,351(49.9)$ \\
\hline \multicolumn{2}{|l|}{ Education level } \\
\hline Less than bachelor's degree & $2,370(50.3)$ \\
\hline Bachelor's degree and above & $2,338(49.6)$ \\
\hline \multicolumn{2}{|l|}{ Self-rated health } \\
\hline Excellent & $1,302(27.6)$ \\
\hline Very good & $1,650(35.0)$ \\
\hline Good & $1,066(22.6)$ \\
\hline Fair & $593(12.6)$ \\
\hline Poor & $98(2.1)$ \\
\hline \multicolumn{2}{|l|}{ Chronic disease present } \\
\hline Yes & $2,575(54.7)$ \\
\hline No & $2,134(45.3)$ \\
\hline \multicolumn{2}{|l|}{ Moderate physical activity } \\
\hline Yes & $784(16.6)$ \\
\hline No & $3,925(83.4)$ \\
\hline \multicolumn{2}{|l|}{ Vigorous physical activity } \\
\hline Yes & $481(10.2)$ \\
\hline No & $4,228(89.8)$ \\
\hline \multicolumn{2}{|l|}{ Nutritional knowledge } \\
\hline Very good & $320(6.8)$ \\
\hline Good & $653(13.9)$ \\
\hline Fair & $851(18.1)$ \\
\hline Poor & $951(20.2)$ \\
\hline Very poor & $1,934(41.1)$ \\
\hline \multicolumn{2}{|l|}{ Sedentary lifestyle } \\
\hline$<2$ h/day & $730(15.5)$ \\
\hline 2 h-4 h/day & $1,212(25.7)$ \\
\hline 4 h-6 h/day & $1,195(25.4)$ \\
\hline$>6$ h/day & $1,572(33.4)$ \\
\hline
\end{tabular}

between weight misperception and BMI, sociodemographic factors, behavioral risk factors, and health status.

\section{Patient and Public Involvement}

No patient involved.

Data Analysis

Descriptive analysis was used to describe the variables. Bivariate analysis $\left(\chi^{2}\right)$ was used to compare categorical data. As the data were collected electronically, there were no missing data. SPSS version 20 was used to analyze the data. 
Table 2. Distribution of BMI categories by age-group, gender, and weight perception

\begin{tabular}{|c|c|c|c|c|c|}
\hline \multicolumn{6}{|l|}{ Gender } \\
\hline Male & $133(5.7)$ & $1,055(44.9)$ & $741(31.5)$ & $422(17.9)$ & 2,351 (49.93) \\
\hline Female & $150(6.4)$ & $917(38.9)$ & $690(29.3)$ & $601(25.5)$ & $2,358(50.07)$ \\
\hline Total & $283(6.0)$ & $1,972(41.9)$ & $1,431(30.4)$ & $1,023(21.7)$ & 4,709 \\
\hline \multicolumn{6}{|l|}{ Age-group, years } \\
\hline $18-19$ & $46(16.3)$ & $130(6.6)$ & $43(3.0)$ & $36(3.5)$ & $255(5.4)$ \\
\hline $30-39$ & $44(15.5)$ & $471(23.9)$ & $311(30.4)$ & $183(17.9)$ & $1,009(21.4)$ \\
\hline $40-49$ & $25(8.8)$ & $322(16.3)$ & $386(27.0)$ & $311(30.4)$ & $1,044(22.2)$ \\
\hline $50-59$ & $11(3.9)$ & $161(8.2)$ & $201(14.0)$ & $182(17.8)$ & $555(11.8)$ \\
\hline+60 & $11(3.9)$ & $86(1.8)$ & $113(7.9)$ & $80(7.8)$ & $290(6.2)$ \\
\hline \multicolumn{6}{|l|}{ Weight misclassification } \\
\hline Properly classified their weight & $186(65.7)$ & $1,303(66.1)$ & $913(63.8)$ & $331(32.4)$ & $2,773(58.0)$ \\
\hline Overweight & $18(6.4)$ & $362(18.4)$ & $913(63.8)$ & $606(59.2)$ & $1,899(40.3)$ \\
\hline Obese & $1(0.4)$ & $16(0.8)$ & $74(5.2)$ & $331(32.4)$ & $422(9.0)$ \\
\hline
\end{tabular}

\section{Results}

\section{Demographics and Response Rate}

Of the 6,239 people contacted via phone, 4,709 (75.5\%) responded and agreed to complete the interview, with equal distribution among the 13 administrative regions of Saudi Arabia. Of the total sample, $50.1 \%$ were female, the mean age was $36.4 \pm 13.5$ (range $=18-90$ ), and $59.6 \%$ had a bachelor's degree or higher (Table 1). The majority (70.1\%) of participants indicated having weighed themselves within the past 30 days.

\section{Obesity Prevalence and Distribution}

Table 2 shows the distribution of BMI by gender, agegroup, and weight perception.

\section{Association between Weight Misperception and Sociodemographic Factors}

Of the total sample, $42.0 \%$ misclassified their weight (Table 2). Age-group was significantly associated with weight misperception, with misperception occurring more frequently in older age-groups, $\chi^{2}(5, N=4,702)=$ $44.13, p<0.001$. Education was also strongly associated with weight misperception, with misperception occurring more frequently in participants who had not earned a bachelor's degree, $\chi^{2}(1, N=4,702)=9.00, p=0.003$. Although there were no significant differences between male and female participants, $\chi^{2}(1, N=4,702)=1.33, p$ $=0.249$, women were slightly more inclined to misclassify their weight $(42.8 \%)$ than men $(41.1 \%)$.

\section{Associations between Weight Misperception and \\ Behavioral Risk Factors}

Sedentary lifestyle was significantly associated with weight misperception, with more sedentary participants more likely to have weight misperception, $\chi^{2}(3, N=$ $4,702)=11.59, p=0.009$. However, neither intensive physical activity, $\chi^{2}(1, N=4,702)=0.32, p=0.569$, nor moderate physical activity, $\chi^{2}(1, N=4,702)=3.03, p=$ 0.081 , was associated with weight misperception. Likewise, nutritional knowledge was not associated with weight perception, $\chi^{2}(4, N=4,702)=7.28, p=0.122$.

\section{Association between Weight Misperception and Health Status}

Misclassification of weight status was related to BMI: $36.2 \%$ of those considered overweight and $67.6 \%$ of those considered obese misclassified their weight status by underestimating their BMI category, with obese participants significantly more likely to misperceive their weight than other BMI groups, $\chi^{2}(4, N=4,702)=353.94, p=<0.001$. Having at least one chronic disease was significantly associated with weight misperception, $\chi^{2}(1, N=4,702)=$ $17.00, p<0.001$, with misperception occurring in $45.2 \%$ 
of participants who had at least one chronic condition. In addition, there was a significant association between selfrated health and weight misperception, $\chi^{2}(4, N=4,702)$ $=15.52, p=0.004$, in which a lower self-rated health was associated with greater weight misperception.

\section{Discussion}

This is the first study to evaluate weight misperception and its correlates among a large national coverage sample of Saudi adults. Our study examined the relationship between weight misperception and sociodemographic factors including sex, age, and educational level; health factors including BMI, diagnosed chronic diseases, and selfrated health; and behavioral factors including physical activity, sedentary behavior, and basic nutritional knowledge. Of the total sample, $42 \%$ of participants did not have an accurate perception of their weight. This misperception was most pronounced in obese participants: $67.6 \%$ of obese participants misclassified their weight compared to $36.2 \%$ of overweight participants, $33.9 \%$ of normal weight participants, and $34.3 \%$ of underweight participants. About $8.4 \%$ of the obese participants in this study perceive themselves to be underweight or normal weight, which is similar to previous studies where the most common misclassification among obese participants was underestimation [25]. In line with Saudi Arabian [21] and international studies (25), our findings indicate that obese individuals are particularly vulnerable to weight misperception.

Weight misperception was not significantly associated with gender, physical activity, or nutritional knowledge but was associated with education, sedentary lifestyle, self-rated health, the presence of a chronic disease, and age. Overall, individuals who misperceived their weight had lower levels of education, a more sedentary lifestyle, lower self-rated health, and one or more diagnosed chronic diseases. These results are broadly consistent with those of previous studies. For example, a study on Saudi female students found that participants with higher levels of education had decreased odds of weight misperception [26]. Likewise, university graduates in Saudi Arabia and the Eastern Caribbean had more realistic weight perceptions than their less educated peers $[20,26]$. Education itself is also a risk (or protective factor) for obesity and a range of healthy behaviors: higher levels of education are associated with lower levels of obesity [27] and more weight loss attempts and healthy weight loss behaviors $[28,29]$. Given the increased vulnerability of indi- viduals with lower levels of education to unhealthy weight behaviors, weight misperception, and subsequent obesity, particular attention and effort should be put into understanding, educating, and working with this demographic.

Individuals who misperceived their weight were also more sedentary, more likely to have 1 or more chronic diseases, and more likely to view themselves as having poorer health. Interestingly, there was no significant association between weight misperception and participating in physical activity, indicating that accurately perceiving oneself as being overweight or obese does not necessarily motivate individuals to participate in physical activity. This is consistent with findings from other studies $[30,31]$.

Similar to previous studies, we observed a significant association between age and weight perception, with older adults more likely to misperceive their weight than their younger peers [25]. Older adults were also more likely to be overweight and obese, which may contribute in part to the observed relationship between age and weight misperception.

While obese participants were more likely to underestimate their weight, about $6.8 \%$ of the participants who are underweight perceive themselves to be overweight. Both cases might be due to the sociocultural factors that may contribute to inaccurate self-perceived weight categorization. These include social group effects, in which individuals who are overweight appear "normal" due to the prevalence of other overweight individuals in the same social environment [32, 33]. For instance, children and adolescents who encounter overweight or obese family members or schoolmates on a regular basis may develop inaccurate perceptions of what constitutes appropriate weight status [34]. This "peer effect" is evident in environments ranging from professional groups, agegroups, cultural groups, and urban/rural settings. Further, as rates of obesity increase, so does the likelihood of inaccurate weight perception. Given the high and increasing rates of obesity in Saudi Arabia, the peer effect is likely to become a growing factor influencing weight perception.

Weight misperception can have far-reaching effects on overall health. Our study demonstrated that individuals who misperceived their weight were more likely to be obese and sedentary and have chronic diseases. Previous research has further shown that people who misclassify their weight are also less likely to have an interest in weight loss, follow diet plans, or engage in physical activity [14, 35-37]. Individuals are unlikely to be receptive to 
discussions of obesity and associated health risks if they do not identify themselves as being obese. Therefore, increasing patient awareness, discussing and correcting weight misperceptions, and ensuring readiness for change are essential prerequisites to any lifestyle intervention for weight loss [38, 39].

\section{Strengths and Limitations}

This is the first study examining weight misperception across the general population in Saudi Arabia at a national level. We included a large sample of adults across the 13 regions of Saudi Arabia and evaluated a range of sociodemographic factors, behavioral risk factors, and health status factors to better understand the correlates of weight misperception. Although a number of significant associations were demonstrated, it is worth noting that the cross-sectional design of our study limits the ability to determine causal relationships.

Our study was based largely on self-reported data, which may have affected the validity of BMI categorizations and consequent levels of misclassification. Previous studies have indicated that using self-reported weight and height data as compared with actual weight and height data can result in lower occurrences of underestimation and higher occurrences of overestimation [40]. The use of objectively measured BMI is preferable as it would provide more accurate estimates of weight misperception. However, we strive to strengthen this study by asking the participants about the last time they measured their weight, which was slightly above $70 \%$ compared with the last month.

We used the proportional quota (nonprobability) sampling technique to ensure that our sample was representative of the general population. This approach provides more statistical power to detect changes at both the national and regional levels and allows data to be stratified by the most affected regions and subpopulations to create a more in-depth picture of the effects of weight misperception. Although this technique carries a risk of selection bias, it was the best available option, given that data were collected during the COVID-19 pandemic. Data integrity checks and the sampling automation inherent to the QPlatform data collection system minimized invalid data entry and prevented human selection bias. Linguistic validation and questionnaire piloting were employed to strengthen the questionnaire's reliability.

\section{Conclusion}

Overall, we found that weight misperception among Saudi adults was significantly associated with obesity, age, education, sedentary lifestyle, self-rated health, and chronic disease. The high prevalence of weight misperception in the present study highlights the importance of screening for weight misperception, addressing weight misperception before undertaking weight loss interventions, and considering interventions that promote healthy perceptions of body image.

\section{Statement of Ethics}

The Ethics Committee of the Sharik Association for Health Research approved this research project (approval No.: 2020-3), according to national research ethics regulations. Consent to participate was obtained verbally during the phone interviews with participants and recorded in the data collection system.

\section{Conflict of Interest Statement}

The authors declare that the research was conducted in the absence of any commercial or financial relationships that could be construed as a potential conflict of interest.

\section{Funding Sources}

We have received no funds to conduct this study.

\section{Author Contributions}

Nora A. Althumiri was involved in the study design, data collection, and writing the final draft; Mada $\mathrm{H}$. Basyouni was involved in the study design and data collection; Nasser F. BinDhim was involved in the study design, data analysis and interpretation, and writing and reviewing the final draft; Saleh A. Alqahtani has involved in reviewing the final draft and approved the final version to be published. N.A.A., M.H.B., N.F.B., and S.A.A. participated in the conceptual design and formulation of the research questions. All of the authors participated in developing and reviewing the manuscript. N.A.A. supervised and managed the data collection process. N.A.A. and N.F.B. analyzed the data. All authors approved the final manuscript.

\section{Data Availability Statement}

The data are available from the Sharik Association for Health Research upon request. 


\section{References}

1 NCD Risk Factor Collaboration (NCD-RisC). Trends in adult body-mass index in 200 countries from 1975 to 2014: a pooled analysis of 1698 population-based measurement studies with 19.2 million participants. Lancet. 2016; 387(10026):1377-96.

2 World Health Organziation. Global health observatory (GHO) data: overweight and obesity. Available from: www.who.int/gho/ ncd/risk_factors/overweight_text/en/.

3 Althumiri NA, Alammari NS, Almubark RA, Alnofal FA, Alkhamis DJ, Alharbi LS, et al. The national survey of health, diet, physical activity and supplements among adults in Saudi Arabia. Fdrsj. 2018;1(1):1.

4 Althumiri NA, Basyouni MH, AlMousa N, AlJuwaysim MF, Almubark RA, BinDhim NF, et al. editors. Obesity in Saudi Arabia in 2020: prevalence, distribution, and its current association with various health conditions. Healthcare; 2021: Multidisciplinary Digital Publishing Institute.

5 BinDhim NF, Althumiri NA, Basyouni $\mathrm{MH}$, AlMousa N, AlJuwaysim MF, Alhakbani A, et al. Exploring the impact of COVID-19 response on population health in Saudi Arabia: results from the "sharik" health indicators surveillance system during 2020. Int J Environ Res Pub Health. 2021;18(10):5291.

6 Kopelman P. Health risks associated with overweight and obesity. Obes Rev. 2007; 8(Suppl 1):13-7.

7 Althumiri NA, Basyouni $\mathrm{MH}$, BinDhim NF. Exploring the association between physical activity and risk of mental health disorders in Saudi Arabian adults: Cross-sectional Study. JMIR Public Health Surveill. 2021;7(4):e25438.

8 Pereira-Miranda E, Costa PRF, Queiroz VAO, Pereira-Santos M, Santana MLP. Overweight and obesity associated with higher depression prevalence in adults: a systematic review and meta-analysis. J Am Coll Nutr. 2017 Mar-Apr;36(3):223-33.

9 Weinberger NA, Kersting A, Riedel-Heller SG, Luck-Sikorski C. Body dissatisfaction in individuals with obesity compared to normalweight individuals: a systematic review and meta-analysis. Obes Facts. 2016;9(6):424-41.

10 Crow S, Eisenberg ME, Story M, NeumarkSztainer D. Psychosocial and behavioral correlates of dieting among overweight and nonoverweight adolescents. J Adolesc Health. 2006;38(5):569-74.

11 Neumark-Sztainer D, Story M, Hannan PJ, Perry CL, Irving LM. Weight-related concerns and behaviors among overweight and nonoverweight adolescents: implications for preventing weight-related disorders. Arch Pediatr Adolesc Med. 2002;156(2):171-8.

12 Yan H, Wu Y, Oniffrey T, Brinkley J, Zhang $\mathrm{R}$, Zhang $\mathrm{X}$, et al. Body weight misperception and its association with unhealthy eating behaviors among adolescents in China. Int J Environ Res Public Health. 2018;15(5):936.

13 Bui AL, Moscoso MG, Bernabe-Ortiz A, Checkley W, Gilman RH, Smeeth L, et al. A sec- ondary analysis examining the concordance of self-perception of weight and actual measurement of body fat percentage: the CRONICAS Cohort Study. BMC Obes. 2019;6(1):9.

14 Duncan DT, Wolin KY, Scharoun-Lee M, Ding EL, Warner ET, Bennett GG. Does perception equal reality? Weight misperception in relation to weight-related attitudes and behaviors among overweight and obese US adults. Int J Behav Nutr Phys Act. 2011;8(1):20.

15 Zaccagni L, Masotti S, Donati R, Mazzoni G, Gualdi-Russo E. Body image and weight perceptions in relation to actual measurements by means of a new index and level of physical activity in Italian university students. J Transl Med. 2014;12(1):42.

16 Robinson E. Overweight but unseen: a review of the underestimation of weight status and a visual normalization theory. Obes Rev. 2017; 18(10):1200-9.

17 Herman KM, Hopman WM, Rosenberg MW. Self-rated health and life satisfaction among Canadian adults: associations of perceived weight status versus BMI. Qual Life Res. 2013; 22(10):2693-705.

18 Burke MA, Heiland FW, Nadler CM. From "overweight" to "about right": evidence of a generational shift in body weight norms. Obesity. 2010;18(6):1226-34

19 Kim S, So WY. Secular trends in the prevalence of weight misperception among Korean adults, 2001-2013. Obes Res Clin Pract. 2018; 12(4):346-50

20 Alyousefi NA, Alqarni FD, Alqarni BS, Almutairi MA, Almazroa SI, Alshehri YM, et al. Association of weight misperception with weight loss attitudes, behaviors and psychological distress in Saudi adult population. Biomed Res. 2017;28(6).

21 BinDhim NF. Smart Health Project. 2012. Available from: https://shproject.net/.

22 Cohen J. Statistical power analysis for the behavioral sciences: Academic press; 2013.

23 Sharik Association for Health Research. Available from: https://sharikhealth.com/.

24 National health and nutrition examination survey centers for disease control and prevention growth charts: United States. Statistics NCfH: US Department of Health and Human Services; 2008. Available from: http://www. cdc.gov/nchs/about/major/nhanes/growthcharts/charts.htm.

25 Freigang R, Geier A-K, Schmid GL, Frese T, Klement A, Unverzagt S. Misclassification of self-reported body mass index categories: a systematic review and meta-analysis. Dtsch Arztebl Int. 2020;117(15).

26 Hassan S, Ojo T, Galusha D, Martinez-Brockman JL, Adams OP, Maharaj R, et al. Obesity and weight misperception among adults in the Eastern Caribbean health outcomes research network (ECHORN) Cohort Study. Obes Sci Pract. 2018;4(4):367-78.

27 Memish ZA, Bcheraoui CE, Tuffaha M, Robinson M, Daoud F, Jaber S, et al. Obesity and associated factors: Kingdom of Saudi Arabia, 2013. Prev Chronic Dis. 2014;11:E174.

28 O'Brien MJ, Whitaker RC, Yu D, Ackermann RT. The comparative efficacy of lifestyle intervention and metformin by educational attainment in the diabetes prevention program. Prev Med. 2015;77:125-30.

29 Kakinami L, Gauvin L, Barnett TA, Paradis G Trying to lose weight: the association of income and age to weight-loss strategies in the US. Am J Prev Med. 2014;46(6):585-92.

30 Xu F, Greaney ML, Cohen SA, Riebe D, Greene GW. The association between adolescent's weight perception and health behaviors: analysis of national health and nutrition examination survey data, 2011-2014. J Obes. 2018;2018:3547856.

31 Southerland J, Wang L, Richards K, Pack R, Slawson DL. On academics misperceptions of overweight: associations of weight misperception with health-related quality of life among normal-weight college students. Public Health Rep. 2013;128(6):562-8.

32 Ver Ploeg ML, Chang HH, Lin BH. Over, under, or about right: misperceptions of body weight among food stamp participants. Obesity. 2008;16(9):2120-5.

33 Opie CA, Glenister K, Wright J. Is social exposure to obesity associated with weight status misperception? Assessing Australians ability to identify overweight and obesity. BMC public health. 2019;19(1):1222.

34 Maximova K, McGrath JJ, Barnett T, O'Loughlin J, Paradis G, Lambert M. Do you see what I see? Weight status misperception and exposure to obesity among children and adolescents. Int J Obes. 2008;32(6):1008-15.

35 Skinner AC, Weinberger M, Mulvaney S, Schlundt D, Rothman RL. Accuracy of perceptions of overweight and relation to self-care behaviors among adolescents with type 2 diabetes and their parents. Diabetes Care. 2008;31(2):227-9.

36 Park S, Lee S, Hwang J, Kwon J-W. The impact of weight misperception on health-related quality of life in Korean adults (KNHANES 2007-2014): a community-based Cross-Sectional Study. BMJ open. 2017;7(6):e016098.

37 Park B, Cho HN, Choi E, Seo DH, Kim S, Park YR, et al. Self-perceptions of body weight status according to age-groups among Korean women: a nationwide population-based survey. PLoS One. 2019;14(1):e0210486.

38 Kuchler F, Variyam JN. Mistakes were made: misperception as a barrier to reducing overweight. Int J Obes Relat Metab Disord. 2003; 27(7):856-61.

39 Aveyard P, Lewis A, Tearne S, Hood K, Christian-Brown A, Adab P, et al. Screening and brief intervention for obesity in primary care: a parallel, two-arm, randomised trial. Lancet. 2016;388(10059):2492-500.

40 Dalton WT III, Wang L, Southerland JL, Schetzina KE, Slawson DL. Self-reported versus actual weight and height data contribute to different weight misperception classifications. South Med J. 2014;107(6):348. 